\title{
DC Voltage Calibrator Based on an Array of High-Temperature Superconductor Josephson Junctions
}

\author{
Alexander M. Klushin, Alexander V. Komkov, Valentina D. Gelikonova, Solomon I. Borovitskii, and M. Siegel
}

\begin{abstract}
We have developed and investigated the main parts of a voltage calibrator with an output voltage up to $10 \mathrm{~V}$. The calibrator is based on a quantum dc voltage which is generated by an array of high-temperature superconductor Josephson junctions. The described concept and the demonstrated parameters of the main parts of the instrument show, that a combined uncertainty of the output voltage of a few parts in $10^{8}$ can be obtained.
\end{abstract}

Index Terms-Comparison, high-temperature superconductors, Josephson array, measurement standard, voltage.

\section{INTRODUCTION}

$\mathbf{V}$ OLTAGE references used in secondary voltage standards, voltage calibrators and digital voltmeters are based on Zener diodes. The specified stability over a one-year period of the $10-\mathrm{V}$ output of the Zener standards is of the order of one part in $10^{6}$ of the nominal output voltage [1]. Using great care and by modeling a temporal drift, pressure, temperature and humidity effects, uncertainties of order of a few parts in $10^{8}$ over periods of months have been claimed [2]. But this uncertainty can be expected only for single instruments with individually measured characteristics [3].

The application of a Josephson reference voltage generated on arrays of high-temperature superconductor (HTS) junctions for the calibration of Zener diodes permits to retain a small uncertainty of the secondary voltage standards over the whole operating period. At the same time, the influence of the parameters mentioned above on the output voltage will be eliminated. Moreover, reference voltages from 0.1 to $10 \mathrm{~V}$ in steps of $0.1 \mathrm{~V}$ can be obtained and used for testing the linearity of precision digital voltmeters with the relative uncertainty of a few parts in $10^{8}$.

\section{Concept of the DC Voltage Calibrator}

The concept of the voltage calibrator is based on the results reported in [4]. In our instrument, the reference voltage of a Zener diode is calibrated against a Josephson voltage $V_{J}$ of 10 to $50 \mathrm{mV}$ generated by the arrays of HTS junctions. To match the output voltage of the Zener diode with the Josephson voltage

Manuscript received June 17, 2002; revised November 4, 2001. This work was supported by German BMBF under Contract 13N7534/4.

A. M. Klushin and M. Siegel are with the Institut für Schichten und Grenzflächen (ISG), Forschungszentrum Jülich $\mathrm{GmbH}$, Jülich, Germany (e-mail: a.klushin@fz-juelich.de).

A. V. Komkov, V. D. Gelikonova, and S. I. Borovitskii are with the Institute of Electronic Measurements KVARZ, Nizhny Novgorod, Russia.

Digital Object Identifier 10.1109/TIM.2003.811581 two equal dividers I and II are used. Divider I is shown in Fig. 1. Both three decade dividers consist of ten-tap foil resistors equal to $R_{0.1}=R_{1}=10 \times 12.5 \Omega$ and $R_{10}=10 \times 125 \Omega$. The thermal coefficient of the resistors does not exceed $10^{-6} \mathrm{~K}^{-1}$. Two stable bias current sources, based on Zener diodes, induce voltage drops $V_{0.1}$ of about $0.1 \mathrm{~V}$ on the divider $R_{0.1}, V_{1}$ of about $1 \mathrm{~V}$ on the divider $R_{1}$, and $V_{10}$ of about $10 \mathrm{~V}$ on the divider $R_{10}$. To decrease the thermal electromotive force (EMF) drift, all of the important parts of the voltage reference (dividers, switches, nanovoltmeter amplifier and two bias current sources) are placed in a temperature-controlled enclosure at the temperature of $35^{\circ} \mathrm{C} \pm 0.05{ }^{\circ} \mathrm{C}$. The stability of the temperature in the period of the calibration $(1 \mathrm{~h})$ was a factor of 10 , which guaranteed a relative thermal variation of the foil resistors smaller than $10^{-8}$. The insulation resistance between the outer and the inner shields, as well as between the shields and the electronic components, was of the order of $10^{11} \Omega$.

The calibration process consists of step-by-step determination of the voltages $V_{0.1}, V_{1}, V_{10}$ and, finally, of the output voltage of the device under test, $V_{\text {DUT }}$. A special switch, not shown in Fig. 1, allows us to make this comparison for two polarities of the bias current. For the determination of $V_{0.1}$ on voltage divider I, shown in Fig. 1, we measured the mean values of the voltage differences between the Josephson voltage $V_{J}$ and voltage drops $V_{0.1, i}^{I}$ on resistors $R_{0.1, i}^{I}(i=1,2, \ldots, p)$ of the divider $R_{0.1}^{I}$ for the plus and minus polarities of the bias current. Due to the design of the divider, the admissible number of the taps $p$ can be equal to $10,5,2$, and 1 . As a result of the measurements we calculate the sum of the mean differences. The desired value of $V_{0.1}$ will be equal to $V_{0.1}=p \cdot V_{J}+\delta_{0.1}$. In an analogous way, voltages $V_{1}, V_{10}$, and $V_{\text {DUT }}$ can be found. For the determination of the voltage $V_{1}$, we measured the sum $\delta_{1}$ of mean values of the voltage differences between voltage $V_{0.1}^{I}$ and voltage drops on resistors $R_{1, i}^{I I}(i=1,2, \ldots, 10)$ of the divider $R_{1}^{I I}$. For the determination of the voltage $V_{10}$, we measured the sum $\delta_{10}$ of mean values of the voltage differences between voltage $V_{1}^{I}$ and voltage drops on resistors $R_{10, i}^{I}(i=1,2, \ldots, 10)$ of the divider $R_{10}^{I}$. Finally, the voltage differences $\delta_{\text {DUT }}$ between $V_{10}$ and the unknown voltage $V_{\text {DUT }}$ are measured. The desired value of the mean voltage $V_{\text {DUT }}$ will be equal to

$$
V_{\mathrm{DUT}}=100\left(p V_{J}+\delta_{0.1}\right)+10 \delta_{1}+\delta_{10}+\delta_{\mathrm{DUT}} .
$$

The corresponding Type A standard uncertainty can be calculated as seen in (2), at the bottom of the next page. 


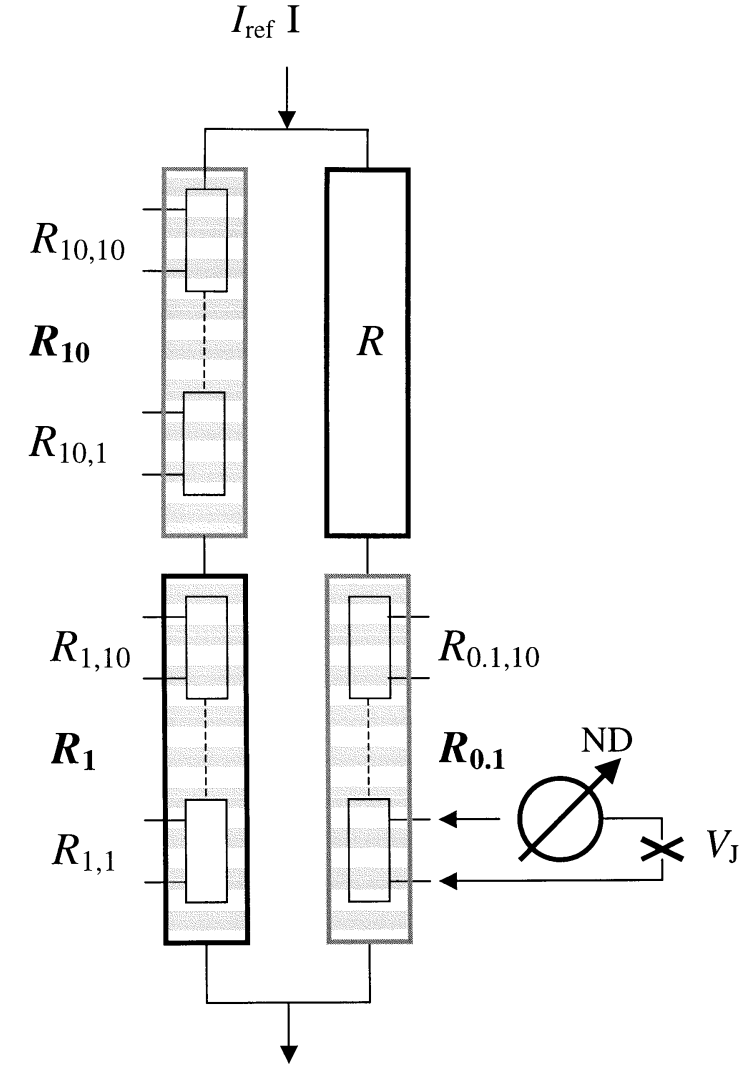

Fig. 1. Basic scheme of the voltage calibrator.

An uncertainty $u_{0.01}$ arises from the calibration of $V_{0.01, i}$ against $V_{J}, u_{0.1}$ arises from the calibration of $V_{1, i}$ against $V_{0.1}$, $u_{1}$ arises from the calibration $V_{10, i}$ against $V_{1}$, and $u_{10}$ arises from the calibration $V_{\text {DUT }}$ against $V_{10}$. All measured uncertainties from $u_{0.01}$ to $u_{10}$ include two components. The first component $u_{n v}$ arises from the noise of the null-detector. The second component $u_{z}$ arising from the noise of the Zener diode limits the relative uncertainty of the voltage calibrator. In the optimal case, the influence of $u_{n v}$ on $u_{\mathrm{DUT}}^{A}$ should be comparable or even smaller than $u_{z}$. The contribution of $u_{n v}$ in $V_{\mathrm{DUT}}$ is more pronounced by the calibration of $V_{0.1}$. For example, for $u_{n v}=0.5 \mathrm{nV}$ the contribution of $u_{0.01}$ to the relative uncertainty of calibration of $V_{\text {DUT }}$ will be about one part in $10^{8}$ for $p$ equal to 10 or 5 , which corresponds to $V_{J}$ equal to $10 \mathrm{mV}$ or $20 \mathrm{mV}$. At a larger $V_{J}$, the influence of $u_{n v}$ on $u_{\text {DUT }}$ can be neglected.

\section{Performance of the Voltage Calibrator}

In this section, we describe the parameters of the arrays of HTS Josephson junctions, frequency synthesizer, nanovoltmeter, and Zener-based current sources.

\section{A. Josephson Reference Voltage Technology}

We use a series array of $\mathrm{HTS} \mathrm{YBa}_{2} \mathrm{Cu}_{3} \mathrm{O}_{7}$ (YBCO) Josephson junctions for generating a reference voltage $V_{J}$ at elevated temperature [5]. Recently, quantum voltages of an array of YBCO bicrystal junctions were calibrated against a programmable Josephson array voltage standard of the Physikalisch-Technische Bundesanstalt [6]. The coincidence of quantum voltages of the array of HTS junctions at $64 \mathrm{~K}$ and of the array of niobium junctions at $4.2 \mathrm{~K}$ was shown within an uncertainty of 1.7 parts in $10^{8}$. This result has shown that arrays of bicrystal junctions are working properly and can be used for high-precision measurements at elevated temperatures.

Two design features of the arrays should be taken into account in their application in a voltage calibrator. First, by using arrays of nonhysteretic junctions and especially HTS junctions control of the metrologically relevant flatness of Shapiro steps, generated on arrays of HTS junctions, is desirable. Two of the many reasons leading to the final slope of the steps are the spread of the array parameters and the thermal fluctuations at elevated temperatures. Moreover, each new cool-down process leads to a slightly different microwave current distribution and the optimum operating conditions have to be determined again. This is also true even for programmable arrays of niobium SNS junctions with very high critical currents [7]. Second, it is necessary to control gain and linearity of the null-detector by using a reference Josephson voltage in the order of $100 \mu \mathrm{V}$ generated with an array.

To meet these requirements, we have used a series array consisting of 254 HTS Josephson junctions divided into two segments containing 128 and 126 junctions, respectively. Shunted bicrystal junctions were fabricated using Au-YBCO bilayers deposited in situ on symmetrical yttrium-stabilized zirconium oxide substrates with a misorientation angle of $19^{\circ}$ or $24^{\circ}$. The thickness of YBCO and Au films were 0.3 and $0.6 \mu \mathrm{m}$, respectively. The arrays were formed by meandering in an Au-YBCO bilayer across the grain boundary. The width of the Josephson junctions was $3 \mu \mathrm{m}$ and the distance between junctions was $1 \mu \mathrm{m}$. The critical current of the array at liquid nitrogen temperature was $I_{c}=0.36 \mathrm{~mA}$. The average resistance for shunted junctions was $R=0.035 \Omega$. Therefore, the minimum characteristic voltage $V_{c}=I_{c} R$ was $12.6 \mu \mathrm{V}$. Each sub-array was biased with a separate dc current source. Fig. 2 shows $I-V$ characteristics of sub-arrays measured in liquid nitrogen under microwave irradiation at the frequency $f=19293 \mathrm{MHz}$. The amplitude of Shapiro steps $\Delta I_{1}$ was about 150 and $180 \mu \mathrm{A}$ for sub-arrays containing 126 and 128 junctions, respectively.

By setting sub-arrays in series opposition, high precision measurements of step flatness can be made. At the same time a voltage difference of about $78 \mu \mathrm{V}$ was used to determine the gain of the nanovoltmeter.

$$
u_{\mathrm{DUT}}^{A}=\sqrt{\left(100 \sqrt{\frac{p}{2}} \cdot u_{0.01}\right)^{2}+\left(10 \sqrt{5} \cdot u_{0.1}\right)^{2}+\left(\sqrt{5} \cdot u_{1}\right)^{2}+\left(\frac{u_{10}}{\sqrt{2}}\right)^{2}}
$$




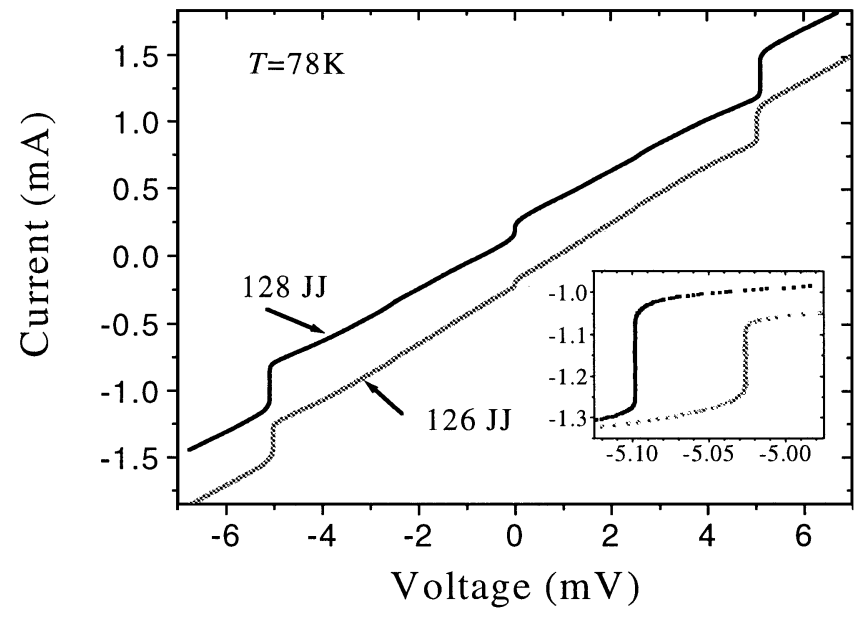

Fig. 2. Current-voltage characteristics of 126 and 128 series connected, shunted YBCO bicrystal junctions at the temperature $T=78 \mathrm{~K}$. Irradiation frequency was $f=19293 \mathrm{MHz}$. For clarity, the curves are shifted by $\pm 0.2 \mathrm{~mA}$. The inset shows an enlarged portion of the curves, demonstrating step width and steepness.

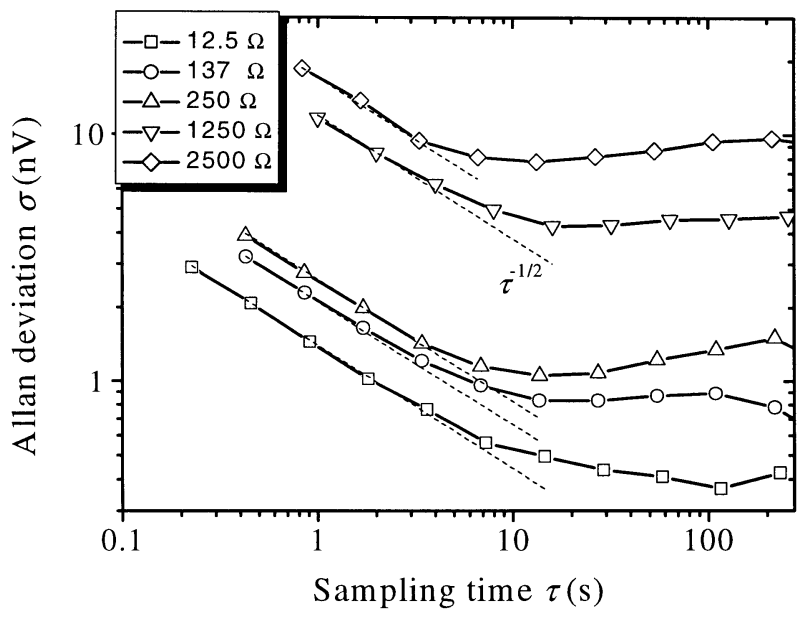

Fig. 3. Allan deviation for EM A22 nanovoltmeter amplifier for different source resistances. Bars represent $1-\sigma$ standard deviations.

\section{B. Frequency Synthesizer}

The phase-locked synthesizer provides microwave power from 15 to $17 \mathrm{dBm}$ in the frequency band from 18 to $20 \mathrm{GHz}$. The output frequency can be tuned in $1 \mathrm{kHz}$ steps. The synthesizer has phase noise of $-82 \mathrm{dBc}$ at a $1 \mathrm{kHz}$ offset from the center frequency. The reference frequency for the synthesizer is obtained from a rubidium frequency standard. We determined the relative uncertainty of the frequency to $2 \times 10^{-10}$.

\section{Null-Detector}

We used an EM Electronics dc amplifier module A22 as a null-detector. Its readings were acquired using an analog-to-digital converter with an integration time over ten power-line cycles. Calibrating the voltage dividers, the source resistance of the amplifier varies from $R_{0.1, i}=12.5 \Omega$ to $2 R_{10}=2500 \Omega$. Fig. 3 shows an Allan deviation $\sigma(\tau)$ [8], [9] for the amplifier A22 with different source resistors and a gain of $3 \times 10^{4}$. The Allan deviation varies nearly as $\tau^{-1 / 2}$ for a source resistance up to $250 \Omega$ and a sampling time $\tau$ between $0.5 \mathrm{~s}$ and $10 \mathrm{~s}$. An
TABLE I

TYPE A UNCERTAINTY COMPONENTS FOR THE CALIBRATION OF THE 10-V VOLTAGE SOURCE

\begin{tabular}{c|c|c|c|c|c|c|c}
\hline \multicolumn{2}{c|}{$u_{0.01}(\mathrm{nV})$} & \multicolumn{2}{c|}{$u_{0.1}(\mathrm{nV})$} & \multicolumn{2}{c|}{$u_{1}(\mathrm{nV})$} & \multicolumn{2}{c}{$u_{D U T}^{A}(\mathrm{nV})$} \\
\hline$u_{\mathrm{nv}}(\mathrm{nV})$ & $u_{\mathrm{z}}(\mathrm{nV})$ & $u_{\mathrm{nn}}(\mathrm{nV})$ & $u_{\mathrm{z}}(\mathrm{nV})$ & $u_{\mathrm{nv}}(\mathrm{nV})$ & $u_{\mathrm{z}}(\mathrm{nV})$ & $u_{\mathrm{nv}}(\mathrm{nV})$ & $u_{\mathrm{z}}(\mathrm{nV})$ \\
\hline 0.5 & 0.1 & 0.8 & 0.7 & 1 & 7 & 5 & 80 \\
\hline \multicolumn{2}{c|}{0.5} & \multicolumn{2}{c|}{1.1} & \multicolumn{2}{c|}{7} & \multicolumn{2}{c|}{80} \\
\hline
\end{tabular}

Allan deviation of $0.5 \mathrm{nV}$ was obtained with a sampling time of $10 \mathrm{~s}$ and a source resistance of $12.5 \Omega$. Recall that this is small enough for the calibration of the $0.1 \mathrm{~V}$ divider with a Type A uncertainty of $1 \times 10^{8}$ for $V_{J}=10 \mathrm{mV}$. The same value of $\sigma(\tau)$ was obtained with a sampling time of 20-30 s, a gain of $10^{2}$ and with an integration time over 50 power-line cycles. At source resistances larger than $1250 \Omega \sigma(\tau)$ showed white noise only when $\tau$ varied approximately from $1 \mathrm{~s}$ to $7 \mathrm{~s}$. For larger sampling times, the Allan deviation became constant near $\sigma(\tau)=8 \mathrm{nV}$ at a source resistance $2500 \Omega$. Such large resistances are used only for the comparison of $10-\mathrm{V}$ dividers, where $8 \mathrm{nV}$ gives a negligibly small uncertainty.

\section{Current Source}

A Zener diode LTZ1000a was used as a voltage reference in a stable current source of about $8.8 \mathrm{~mA}$. We measured the Allan deviation as a function of the voltage difference between $V_{10}$ and a $10 \mathrm{~V}$ output of an electronic standard Fluke model $732 \mathrm{~A}$. An Allan deviation equal to $0.12 \mu \mathrm{V}$ was obtained in $7 \mathrm{~s}$, which limited the Type A standard uncertainty of the new voltage calibrator.

\section{UNCERTAINTY EVALUATION}

Type A uncertainties due to calibration of a voltage $V_{\text {DUT }}$ can be evaluated by using the expression (2). Table I list the sources of these uncertainties and their expected values, which are based on the data described above. A type A uncertainty of $200 \mathrm{nV}$ can be achieved for $V_{J}=10 \mathrm{mV}$.

The type B uncertainty includes many more sources. We suppose the following sources are of primary concern: leakage-current effects, drift of the bias currents during calibration, instability of EMF in the switches required to connect the null-detector to the different parts of the divider, drift of the temperature in the thermostat, divider ratios, amplifier gain nonlinearity, and offset voltages. It is very difficult to take into account the influence of all these sources.

Nevertheless, the presence of two dividers makes it possible to exclude the results with significant systematic uncertainty by the developed procedure of self-calibration. For example, voltage differences between $V_{1}^{I}$ and $V_{1}^{I I}$ of dividers I and II can be found in two ways. First, we measure the difference $\Delta V_{1}^{\prime}=$ $V_{1}^{I}-V_{1}^{I I}$ directly. Second, we find the same difference in three steps: 1) we measure the sum $\delta_{1}^{I}$ of mean values of the voltage differences between voltage $V_{0.1}^{I I}$ and voltage drops on resistors $R_{1, i}^{I}(i=1,2, \ldots, 10)$ of the divider $\left.R_{1}^{I} ; 2\right)$ we measure the sum $\delta_{1}^{I I}$ of mean values of the voltage differences between voltage $V_{0.1}^{I}$ and voltage drops on resistors $R_{1, i}^{I I}(i=1,2, \ldots, 10)$ of the divider $\left.R_{1}^{I I} ; 3\right)$ we measure the difference $\Delta V_{0.1}=V_{0.1}^{I}-V_{0.1}^{I I}$ directly. Steps 1) -3 ) allow us to calculate the value $\Delta V_{1}^{\prime \prime}=$ 
TABLE II

RESUlts of SElF-CALIBRATION OF 1-V AND 10-V DividERS

\begin{tabular}{|c|c|c|c|c|c|}
\hline Run No. & Voltage & Date & $\Delta_{1}(\mathrm{nV})$ & $\Delta_{10}(\mathrm{nV})$ & $u(\mathrm{nV})$ \\
\hline 1 & \multirow[t]{8}{*}{$1 \mathrm{~V}$} & $29.05 .02 \_1$ & 61 & & \pm 18 \\
\hline 2 & & 29.05.02_2 & 43 & & \pm 18 \\
\hline 3 & & $29.05 .02 \_3$ & 56 & & \pm 18 \\
\hline 4 & & $29.05 .02 \_4$ & 27 & & \pm 18 \\
\hline 5 & & $29.05 .02 \_5$ & 6 & & \pm 15 \\
\hline 6 & & $29.05 .02 \_6$ & 90 & & \pm 18 \\
\hline 7 & & $29.05 .02 \_7$ & 10 & & \pm 15 \\
\hline 8 & & 01.06 .02 & 25 & & \pm 18 \\
\hline 9 & \multirow[t]{3}{*}{$10 \mathrm{~V}$} & 24.05 .02 & & -190 & \pm 249 \\
\hline 10 & & 29.05 .02 & & -206 & \pm 276 \\
\hline 11 & & 30.05 .02 & & -162 & \pm 144 \\
\hline
\end{tabular}

$10 \Delta V_{0.1}+\delta_{1}^{I}-\delta_{1}^{I I}$. The difference $\Delta_{1}=\Delta V_{1}^{\prime}-\Delta V_{1}^{\prime \prime}$ and corresponding uncertainties $u(k=3)$ are listed in Table II. Similarly, calculated differences $\Delta_{10}=\Delta V_{10}^{\prime}-\Delta V_{10}^{\prime \prime}$ for $10 \mathrm{~V}$ and corresponding uncertainties are shown in the same table. If the values $\Delta_{1}$ or $\Delta_{10}$ are smaller than $\pm u$, we can suppose that no significant errors have occurred. Table II documents that in the measurements from 1 to 3 and six voltage differences were larger than a $k=3$ uncertainty interval having a level of confidence of approximately 99\%. All other results of self-calibration can be accepted. They show that the relative uncertainty of the self-calibration was approximately $2 \times 10^{-8}$.

\section{CONCLUSION}

We have presented a laboratory prototype of a voltage calibrator, in which a Zener reference voltage can be calibrated against a quantum voltage generated by an HTS array of Josephson junctions. Stable voltages up to $10 \mathrm{~V}$ in steps of $0.1 \mathrm{~V}$ can be obtained with an uncertainty equal to a few parts in $10^{8}$. A link between Josephson standard and Zener reference permits to measure the influence of pressure, temperature and humidity on the output voltage of the electronic standard. Also, it helps to overcome the problem of the deviation of the Zener voltage from the linear drift model, which contributes to a significant part to the uncertainty in comparisons [1]. The suggested method of self-calibration admits control of the systematic uncertainties in the calibration process.

\section{ACKNOWLEDGMENT}

The authors would like to thank O. A. Chevtchenko, A. S. Katkov, F. Piquemal, and T. Witt for helpful discussions.

\section{REFERENCES}

[1] 732B/734A DC Reference Standard Instruction Manual Rev. 3, 11/97, Fluke Corporation, 1997

[2] D. Reymann, T. J. Witt, P. Vrabcek, Y.-H. Tang, C. A. Hamilton, A. S. Katkov, B. Jeanneret, and O. Power, "Recent developments in BIPM voltage standard comparison," IEEE Trans. Instrum. Meas., vol. 50, pp. 207-209, Apr. 2001.

[3] T. J. Witt, "Maintenance and dissemination of representations of the Volt by Zener diode-based dc voltage references," in Proc. BEMC, Harrogate, U.K., 2001.
[4] K. A. Ainitdinov, S. I. Borovitskii, V. Bykov, Y. M. Gryasnov, A. V. Komkov, and V. F. Resinskii, "Industrial voltage standard based on stable point Josephson contact with relative accuracy $1 \times 10^{-7}$," Electron. Meas. KVARZ, no. 3, pp. 5-8, July 1994.

[5] A. M. Klushin, W. Prusseit, E. Sodtke, S. I. Borovitskii, L. Amatuni, and H. Kohlstedt, "Shunted bicrystal Josephson junctions arrays for voltage standard," Appl. Phys. Lett., vol. 69, pp. 1634-1637, Sept. 1996.

[6] A. M. Klushin, R. Behr, K. Numssen, M. Siegel, and J. Niemeyer, "Accurate measurements of quantum voltage steps on arrays of bicrystal Josephson junctions," Appl. Phys. Lett., vol. 80, pp. 1972-1974, Mar. 2002.

[7] B. Jeanneret, A. Rüfenacht, and J. Burroughs, "High precision comparison between SNS und SIS Josephson voltage standards," IEEE Trans. Instrum. Meas., vol. 50, pp. 188-191, Apr. 2001.

[8] D. W. Allan, "Should the classical variance be used as a basic measure in standards metrology?," IEEE Trans. Instrum. Meas., vol. IM-36, pp. 646-654, Apr. 1987.

[9] T. J. Witt, "Using the Allan variance and power spectral density to characterize dc nanovoltmeters," IEEE Trans. Instrum. Meas., vol. 50, pp. 445-448, Apr. 2001

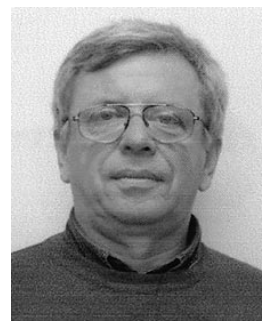

Alexander M. Klushin was born in Gorky, USSR, on January 29, 1946. He received the Dipl.-Radiophys. degree and the Cand. Sci. degree in physics and mathematics from Gorky State University in 1969 and 1985, respectively.

He joined the Institute of Electronic Measurement KVARZ, Nizhny Novgorod, Russia, in 1969. Since 1993, he has been with the Research Center, Jülich, Germany. His current research interest is in the work with arrays of high- $T_{c}$ Josephson junctions for applications in quantum voltage metrology. He has authored 54 publications and holds six patents. received the Dipl.-Radiophys. degree from Gorky State University, Gorky, in 1969.

In 1969, he joined the Institute of Electronic Measurement KVARZ, Nizhny Novgorod, Russia. His scientific interest is in cryoelectronics.

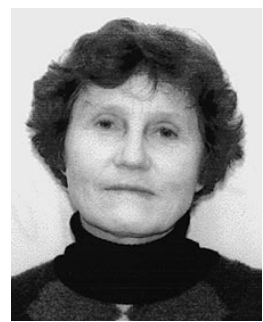

Valentina D. Gelikonova was born in Brjansk, USSR, on January 2, 1947. She received the Dipl.-Radiophys. degree from Gorky State University, Gorky, USSR, in 1969.

In 1969. she joined the Institute of Electronic Measurement KVARZ, Nizhny Novgorod, Russia. Her scientific interest is in cryoelectronics.

Solomon I. Borovitskii was born on September 25, 1921. He received the degree of Candidate of Sciences in physics and mathematics from Gorky State University, Gorky, USSR, in 1950.

His main scientific interest is in cryoelectronics.

M. Siegel, photograph and biography not available at the time of publication. 